\title{
Frecuencia del intercambio de cromátides hermanas en un grupo de individuos expuestos ocupacionalmente a los vapores de derivados del petróleo
}

\author{
Guzmán M.
}

\begin{abstract}
Resumen
La frecuencia de los intercambios de cromátides hermanas (XICH) fue establecida en 156 individuos expuestos ocupacionalmente a los vapores de derivados del petróleo y en 36 individuos ocupacionalmente no expuestos. El grupo expuesto se subdividió en 4 grupos de acuerdo con el área de trabajo. Se encontró una $\mathrm{XICH}$ altamente significativa $(P<0,01)$ en los cuatro grupos de individuos expuestos de las áreas $G 1, P A$, PB y LI $(9,51,9,66,10,20$ y 9,38 , respectivamente), comparado con el grupo control $(7,74)$. Se encontró que tanto la antigüedad laboral como el hábito de fumar y las condiciones intrínsecas de cada área de trabajo, tuvieron efecto sobre la inducción de $\mathrm{ICH}$. La edad de los individuos no influyó significativamente sobre los $\mathrm{ICH}$. Se encontró una reducción significativa $(\mathrm{P}<0,05)$ del índice de cinética celular $(\mathrm{ICC})$ en un grupo de 73 individuos ocupacionalmente expuestos $(\mathrm{ICC}=2,08)$ con relación al grupo de individuos control $(I C C=2,37)$. Los resultados sugieren que la exposición crónica y/o aguda a los vapores de productos de derivados del petróleo inducen la formación de $\mathrm{ICH}$ y podrían causar reducción del ciclo y proliferación celular in vitro en algunos de los individuos expuestos ocupacionalmente a los vapores de derivados del petróleo, dependiendo de la susceptibilidad de cada genoma expuesto.
\end{abstract}

\section{Summary}

Sister chromatid exchange frequencies (XSCE) were evaluated in 156 individuals occupationally exposed to petroleum-vapour derivatives and in 36 non-occupationally exposed individuals. The exposed group was subdivided into four with regard to the work area. A high XSCE $(P, 0.01)$ was found in the four exposed groups, $G 1, P A, P B$ and LI $(9.51,9.66,10.20,9.38$, respectivelly), compared to the control group (7.74). It was found that the total work-exposed time (duration of employment), smoking habits and intrinsic work-area environment, induce SCE. Age did not affect SCE induction. A significant reduction $(\mathrm{P}<0.05)$ in the celullar kinectics' index $(\mathrm{ICK})$ of 73 exposed individuals $(I C K=2.08)$ compared to 27 control individuals (ICK=2.37) was found. The results suggest that chronic and/or acute exposure to petroleum vapour derivatives induce SCE and also could reduce the proliferation and reduction of the cellular cycle in vitro of some of the occupationally exposed individuals, with regard to work-area, total work-exposure time and smoking habits, the susceptibility depending on the degree of radiation to which each genome was exposed.

Facultad de Medicina, Instituto de Biotecnología, Universidad Nacional de Colombia. 
En los humanos, la exposición ocupacional a los solventes orgánicos y/o productos derivados del petróleo se ha asociado con rupturas y rearregios cromosómicos en linfocitos de sangre periférica (1-3) y la exposición al benceno con el desarrollo de cáncer (8-10), especialmente de leucemia mieloide aguda (LMA) (9-11). Estudios realizados en médula ósea de trabajadores expuestos a benceno y de animales experimen tales han mostrado que la exposición aguda a este compuesto produce depresión del sistema nervioso central, dependiente de la dosis, mientras que la exposición crónica puede conducir a pancitopenia o anemia aplástica con dosis mayores de 30 ppm $\mathrm{y}$, en algunos casos, al desarrollo de leucemia con dosis mayores de $100 \mathrm{ppm}$ (8-10). En ratones, Maltoni et al. (11) demostraron que el benceno es un carcinógeno multipotencial y otros estudios concluyen que son los metabolitos fenólicos de este compuesto los responsables de los efectos cito y genotóxicos (12-14). Después de una apropiada activación metabólica in vitro, el benceno causa aumento del intercambio de cromátides hermanas $(\mathrm{ICH})$ y retraso de la división celular de linfocitos humanos en cultivo $(12,15)$. También, el benceno inhibe la proliferación celular de la médula ósea de ratones expuestos por inhalación a este compuesto (16). Los informes sobre lainducción de ICHen linfocitos de sangre periférica por la exposición de humanos al benceno o a otros solventes orgánicos son escasos y contradictorios. Salamanca, Gómez et al. (17) y Exeron et al. (18) encontraron diferencias significativas en la frecuencia de $\mathrm{ICH}$, en un grupo de inhaladores de pegantes a base de benceno y en trabajadores ocupacionalmente expuestos a este compuesto, respectivamente. Otros estudios no informan diferencias significativas (19-23). Portanto, un punto de interés sigue siendo si estos compuestos son mutagénicos y/o carcinogénicos en trabajadores expuestos ocupacionalmente dentro de las concentraciones límites técnicamente aceptadas $(8,24)$.

En el presente trabajo se evaluó el efecto de la exposición ocupacional a los vapores de compuestos derivados del petróleo utilizando la prueba de $\mathrm{ICH}(25)$ considerada como una prueba sensible para detectar mutágenos carcinógenos ambientales (26) y para el estudio de la cinética celular $(12,15,27)$.

\section{Materiales y métodos}

Muestra de estudio: se analizaron 156 trabajadores expuestos a vapores de productos derivados del petróleo. En la tabla 1 se presenta la distribución de estos individuos en cuanto al área de trabajo y se relaciona con la edad, la antigüedad laboral y el hábito de fumar. Se presenta la información del grupo control (individuos no expuestos ocupacionaimente). Todos los trabajadores realizan rotaciones periódicas dentro de su misma área de trabajo. El espacio de trabajo es abierto en las áreas G1, PA y PB y cerrado en LI. Las plantas funcionan con un sistema cerrado de tubería y el riesgo de exposición se presenta por los empaques de las válvulas y en la toma de muestras. En general, los trabajadores utilizan protección con filtros, máscaras y guantes. No se dispuso de información sobre valoraciones atmosféricas de los compuestos. Las muestras de sangre periférica se mantuvieron a $4^{\circ} \mathrm{C}$ y se cultivaron dentro de las primeras 24 horas.

\section{Técnica de $\mathrm{ICH}$}

El análisis cromosómico se realizó sobre cultivos de sangre periférica (28). En resumen, se utilizaron $0,25 \mathrm{ml}$ de sangre venosa heparinizada y se cultivaron durante $72 \mathrm{~h}$ a $37^{\circ} \mathrm{C}$ en $5 \mathrm{ml}$ de medio de cultivo RPMI 1640 (Sigma) con antibióticos

Tabla 1. Muestra de estudio. Distribución por área de trabajo, edad, antigüedad, hábito de fumar.

\begin{tabular}{|c|c|c|c|c|c|}
\hline & G1 & PA & PB & LI & $\mathrm{GC}$ \\
\hline Número de individuos & 47 & 35 & 39 & 35 & 36 \\
\hline Edad promedio (años) & 38 & 34 & 34 & 33 & 33 \\
\hline Rango de edad (años) & : 25-63 & $23-46$ & $23-47$ & $24-49$ & $24-46$ \\
\hline Antigüedad promedio & $: 13,5$ & 10,0 & 11,0 & 11,0 & 0 \\
\hline Rango antigüedad (años) & : $1-31$ & $1-18$ & $1-19$ & $1-26$ & 0 \\
\hline Individuos fumadores & 12 & 10 & 7 & 8 & 5 \\
\hline Células analizadas & $: 1.410$ & 1.192 & 1.143 & 1.258 & 1.045 \\
\hline
\end{tabular}

G1: planta de refinación de materias primas (producción de nafta)

PA: planta de aromáticos (producción de hidrocarburos aromáticos)

PB: planta de balance (producción de gas metano y coke)

LI: laboratorio industrial (control de calidad)

GC: grupo control (individuos no expuestos ocupacionalmente) 
(50 $\mu \mathrm{g}$ de penicilina y $50 \mathrm{ml}$ de estreptomicina), suplementado con $10 \%$ de suero bovino fetal y $0,1 \%$ de fitohemaglutinina $\mathrm{P}$ (Gipco). Durante las últimas $48 \mathrm{~h}$ de cultivo, se adicionaron $4 \mu \mathrm{g} / \mathrm{ml}$ de 5-bromo-2-deoxiuridina (Sigma) y se mantuvieron en la oscuridad. Se adicionó $0,1 \mu \mathrm{g} / \mathrm{ml}$ de Colcemid (Sigma) 20 minutos antes de la recolección. Se utilizó $\mathrm{KCL}(0,075 \mathrm{M})$ como solución y como fijador se utilizó metanol: ácido acético (3:1). Las extensiones se secaron al aire y se procesaron para la visualización de $\mathrm{ICH}$ siguiendo la técnica de fluorescencia más giemsa (FPG) (27).

\section{Análisis estadístico}

1. Frecuencia del intercambio de cromátides hermanas $(\mathrm{XICH})$. Para cada individuo se obtuvo una $\mathrm{XICH}$ sobre 30 metafases analizadas en segunda división celular. Se determinó la $\mathrm{XICH}$ de cada grupo de individuos expuestos (G1, PA, PB, LI) y del grupo control (GC). Se realizaron tres tipos de análisis teniendo en cuenta la antigüedad, la edad y el hábito de fumar. En el primero, el grupo de individuos de cada área de trabajo G1, PA, PB y LI se subdividió arbitrariamente en dos de acuerdo con la antigüedad: entre 1 a 10 años y más de 11 años. En el segundo, el grupo de individuos de cada área de trabajo G1, PA, PB y LI se subdividió en dos de acuerdo con la edad: menores de 35 años y mayores de 36 años. En el tercer análisis, se compararon los indi- viduos fumadores y no fumadores de los grupos de individuos ocupacionalmente expuestos y del grupo control. Los análisis estadísticos se realizaron con un análisis de varianza de una cola y la prueba de Fisher (F-test).

2. Indice de cinética celular. La cinética celular se analizó en 36 individuos del grupo expuesto (PA), 37 individuos del grupo expuesto (LI) y 27 individuos del grupo control (GC). Se analizaron 80 células por individuo y se catalogaron como células de primera, segunda y tercera o más generaciones dependiendo de la tinción diferencial de los cromosomas, tinción homogénea, tinción en arlequín y tinción asimétrica, respectivamente. Se estableció el porcentaje de células en cada división celular y se determinó el índice de cinética celular $(\mathrm{ICC}=1 \times \mathrm{M} 1+2 \times \mathrm{M} 2+3 \times \mathrm{M} 3 / \mathrm{M} 1+\mathrm{M} 2+\mathrm{M} 3)(22)$. Para el análisis estadístico se utilizó la prueba de t Student.

\section{Resultados}

En la figura 1 (b) se muestran los ICH en la célula de segunda división cuyos cromosomas tienen cromátides con tinción en arlequín, blanco y negro. Los $\mathrm{ICH}$ se observan en los sitios de cambio de coloración dentro de un mismo cromosoma. En la tabla 2 se muestra la XICH de cada grupo expuesto y del grupo control. En los grupos G1, PA, PB, LI y GC se encontraron XICH

Tabla 2. Frecuencia de $\mathrm{ICH}$ en los grupos expuestos y grupo control. $\mathrm{ICH}$ con relación a la antigüedad.

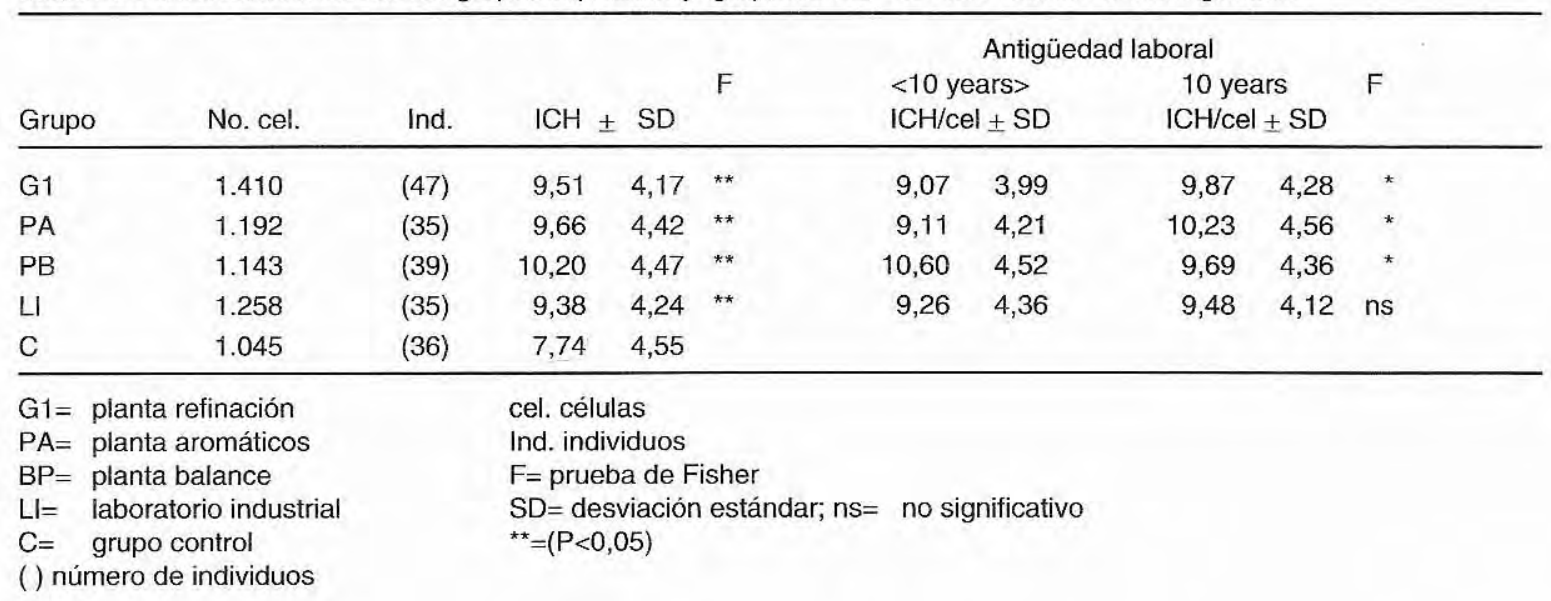


de $9,51,9,66,10,20,9,38$ y 7,74 , respectivamente.Se encontraron diferencias altamente significativas $(P<0,01)$ en la comparación de cada grupo expuesto con el grupo control. En la tabla 2 también se muestra la $\mathrm{XICH}$ de cada grupo expuesto con relación a la antigüedad laboral, individuos con menos o más de 10 años laborales. La comparación de $\mathrm{XICH}$ dentro de cada grupo expuesto $(\mathrm{G} 1=9,07$ vs 9,$87 ; \mathrm{PA}=9,11$ vs $10,23 ; \mathrm{PB}=10,60$ vs 9,69$)$ mostró diferencias significativas $(\mathrm{P}<0,05)$ mientras que las $\mathrm{XICH}$ del grupo LI no se afectaron significativamente por la antigüedad ( $\mathrm{LI}=9,26$ vs 9,48). La edad de los individuos expuestos y controles, menores y mayores de 35 años, no influyó significativamente la $\mathrm{XICH}$ (los resultados no se presentan). En la tabla 3 se muestra la XICH con relación al hábito de fumar, fumadores y no fumadores, de cada grupo expuesto $(\mathrm{G} 1=9,71$ vs 9,$44 ; \mathrm{PA}=9,58$ vs 9,$69 ; \mathrm{PB}=11,18$ vs 9,99 y $\mathrm{LI}=9,74$ vs 9,28 ) del grupo total de individuos expuestos (GTE $=10,05$ vs 9,60$)$ y del grupo control ( $\mathrm{GC}=9,60$ vs 7,64$)$. Se encontró un aumento altamente significativo $(P<0,01)$ de los fumadores vs no fumadores tanto en los grupos expuestos como en el grupo control. $\mathrm{La} \mathrm{XICH}$ de fumadores del grupo control $(9,60)$ tiende a ser mayor que la $\mathrm{XICH}$ de los grupos expuestos no fumadores G1 $(9,44)$ y LI $(9,28)$ y similar a la del grupo PA, tanto de fumadores $(9,58)$ como de no fumadores $(9,69)$.

Tabla 3. Frecuencia de $\mathrm{ICH}$ en individuos expuestos y control con relación al hábito de fumar.

\begin{tabular}{lrrrrrrrr}
\hline $\begin{array}{c}\text { Grupo } \\
\text { ICH/CEL } \pm \text { SD }\end{array}$ & $\begin{array}{c}\text { fumador } \\
\text { ICHD }\end{array}$ & \multicolumn{2}{c}{$\begin{array}{c}\text { No fumador } \\
\text { ICH/CEL } \pm \text { SD }\end{array}$} & IND & $\begin{array}{c}\text { FISHERS' } \\
\text { TEST }\end{array}$ \\
\hline G1 & 9,71 & 4,55 & $(12)$ & 9,44 & 4,04 & $(35)$ & \\
PA & 9,58 & 4,12 & $(10)$ & 9,69 & 4,53 & $(25)$ & \\
PB & 11,18 & 5,13 & $(7)$ & 9,99 & 4,27 & $(32)$ & \\
LI & 9,74 & 4,51 & $(8)$ & 9,28 & 4,00 & $(27)$ & \\
C & 9,60 & 4,00 & $(5)$ & 7,64 & 5,00 & $(31)$ & $* *$ \\
TE & 10,05 & 4,60 & $(42)$ & 9,60 & 4,24 & $(114)$ & $* *$ \\
\hline
\end{tabular}

$\mathrm{TE}=$ grupo total de expuestos

$\mathrm{IND}=$ individuos

$\mathrm{SD}=$ desviación estándar

$* * \quad(P<0,01)$
En el grupo PA, los 25 individuos no fumadores presentaron una $\mathrm{XICH}$ levemente aumentada $(9,69)$ sobre los 10 individuos fumadores $(9,58)$. Sin embargo, en estos casos particulares no se realizaron pruebas de significancia. Los 7 fumadores del grupo $\mathrm{PB}(11,18)$ presentaron la $\mathrm{XICH}$ más alta de todos los grupos de fumadores analizados. En la figura 1 (a, b, c) se muestra cromosomas de células de primera, segunda y tercera división celular, respectivamente. La clasificación de las divisiones celulares se realizó por la tinción típica de los cromosomas en cada una de ellos. En la tabla 4 se muestran los resultados del análisis de la cinética celular. En el grupo de 73 individuos expuestos (PA+LI) el ICC fue de 2,08 y en el grupo 27 controles fue de 2,37 , mostrando diferencias significativas $(P<0,05)$. La comparación entre individuos expuestos y controles, de los porcentajes de células en primera generación $(34,24 \%$ vs $15,38 \%)$ y terceras generaciones $(28,53 \%$ vs $53,20 \%)$, mostró diferencias altamente significativas $(P<0,01)$. La comparación de los porcentajes de células en segunda generación $(34,66 \%$ vs $31,26 \%)$ mostró diferencias significativas $(P<0,05)$.

\section{Discusión}

En el presente estudio se encontró que los cuatro grupos de individuos expuestos ocupacionalmente a los vapores de productos derivados del

Tabla 4. Indice de cinética celular (ICC) y porcentajes celulares en el grupo expuesto y control.

\begin{tabular}{|c|c|c|c|c|c|c|c|}
\hline Grupo & CELL & IND & $\mathrm{ICC}$ & t Student & $\%$ CPD & $\%$ CSD & $\%$ CTD \\
\hline $\mathrm{PA}+\mathrm{LI}$ & 5.000 & (73) & 2,08 & * & 34,24 & 34,66 & 28,53 \\
\hline c & 2,300 & $(27)$ & 2,37 & & 15,83 & 31,26 & 53,20 \\
\hline \multicolumn{8}{|c|}{$\mathrm{PA}+\mathrm{LI}=$ planta aromáticos + laboratorio industrial } \\
\hline \multicolumn{8}{|c|}{$\mathrm{C}=$ grupo control } \\
\hline \multicolumn{8}{|c|}{ Ind= individuos } \\
\hline \multicolumn{8}{|c|}{${ }^{*}=\mathrm{P}<0,050$} \\
\hline \multicolumn{8}{|c|}{$\mathrm{ICC}=$ índice de cinética celular } \\
\hline \multicolumn{8}{|c|}{$\mathrm{CPD}=$ células de primera división } \\
\hline \multicolumn{8}{|c|}{$\mathrm{CSD}=$ células de segunda división } \\
\hline $\mathrm{CTD}=$ & élulas & ter & $\mathrm{ra}$ & isión & & & \\
\hline
\end{tabular}




\section{CINETICA CELULAR}

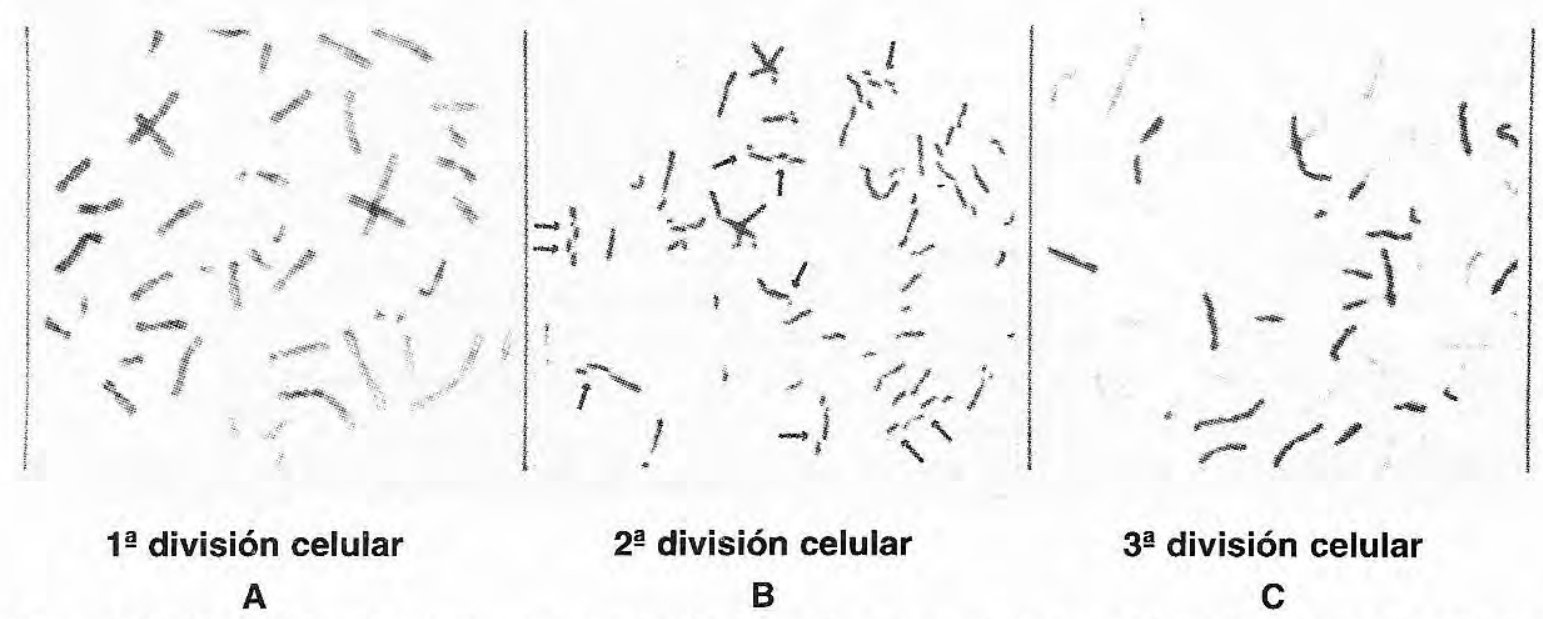

Figura 1. Cinética celular: a) cromosomas con tinción homogénea provenientes de células en primera división; b) cromosomas con tinción simétrica en arlequín provenientes de células en segunda división. Las flechas señalan intercambio de cromátides hermanas $(\mathrm{ICH}) ; \mathrm{c}$ ) cromosomas con tinción asimétrica provenientes de células en tercera división.

petróleo, G1, PA, PB y LI, presentaron una $\mathrm{XICH}$ de $9,51,9,66,10,20$ y 9,38 , respectivamente, significativamente más alta $(\mathrm{P}<0,01)$ que el grupo control de individuos no expuestos ocupacionalmente $(7,74)$. Se encontró un efecto de la antigüedad laboral en las $\mathrm{XICH}$. Las $\mathrm{XICH}$ de los grupos G1, PA y LI están afectadas por los individuos con más de 10 años de antigüedad $(9,87,10,23$ y 9,48$)$ respectivamente. Es importante resaltar que los individuos PA tienen un alto riesgo de exposición a los hidrocarburos aromáticos incluido el benceno. Sin embargo, dada la metodología utilizada no se puede establecer una correlación directa con un compuesto específico. En el área PB, se utilizan fondos de vacío para la producción de gases metano, propano y también coke. En esta planta, los individuos pueden estar expuestos también a metales pesados y otros compuestos. La XICH en la planta PB fue alta $(10,20)$ y se afectó principalmente por los individuos con menos de 10 años de antigüedad $(10,60)$ sobre los de individuos de más de 10 años $(9,69)$. Estos resultados sugieren que en el área PB los individuos con menos de 10 años de antigüedad podrían estar expuestos a concentraciones más altas de algunos compuestos, o expuestos a un mayor riesgo de exposiciones agudas. Se ha informado que los metales pesados podrían ser causa de mayor frecuencia de aberraciones cromosómicas (29). De otra parte, podría ser que los 7 fumadores del grupo $\mathrm{PB}(\mathrm{XICH}$ $=11,08)$, pertenezcan al grupo de individuos con menos de 10 años de antigüedad laboral. Al igual que en otros reportes de la literatura (29-31) se encontró que el cigarrillo es un inductor de ICH. El conjunto de fumadores del grupo de individuos expuestos $(10,05)$ y del grupo control $(9,60)$ presentaron aumento altamente significativo $(\mathrm{P}<0,01)$ de la $\mathrm{XICH}$. Sin embargo, en el área de trabajo PA no se encontraron diferencias significativas entre 10 individuos fumadores $(9,58)$ y 25 individuos no fumadores $(9,69)$. Es decir, que en algunos individuos las $\mathrm{XICH}$ altas podrían deberse al efecto de un conjunto complejo de factores, sin efectos importantes del cigarrillo, mientras que en otros individuos el cigarrillo podría actuar sinergísticamente como lo plantea Skyberg et al. (24). Sin embargo, no se realizó un análisis de multivarianza para establecer la estricta dependencia de cada variable sobre la $\mathrm{XICH}$. Es interesante anotar que entre el $66 \%$ y el $75 \%$ de los $\mathrm{ICH}$ se localizan en los mismos puntos cromosó- 
micos de algunos sitios frágiles $(31,32)$ y algunos sitios frágiles podrían ser base de rupturas y rearreglos cromosómicos (32 - 35). De otra parte, no se encontró efecto de la edad sobre la XICH, al comparar en cada grupo mayores y menores de 35 años. En la literatura los informes sobre el efecto de la edad como inductor de $\mathrm{ICH}$ son contradictorios $(30,36)$. El rango de $\mathrm{XICH}$ dentro de cada grupo expuesto y control, mostró amplias diferencias interindividuales $(6,21$ a 16,74 y 5,78 a $11,8)$. Es decir que, además de la influencia de los factores medioambientales, se debe tener en cuenta que hay susceptibilidades genómicas diferentes al estrés medioambiental, (34). El análisis de cinética celular mostró una reducción significativa $(P<0,05)$ cuando se comparó el grupo de individuos expuestos $(I C C=2,08)$ con el grupo control $(\mathrm{ICC}=2,37)$. Se encontraron diferencias altamente significativas $(P<0,01)$ en la comparación del porcentaje de células en primera, segunda y tercera o más generaciones. En el grupo control el porcentaje de células fue 15,85\%, $31,26 \%$ y $53,20 \%$ para primeras, segundas y terceras o más generaciones y en el grupo expuesto la progresión fue lineal para primeras y segundas generaciones con disminución de terceras generaciones $(34,24 \% ; 34,66 \% ; 28,53 \%)$ respectivamente. Los resultados indican que el tiempo del ciclo celular es más largo en el grupo de individuos expuestos, con reducción de la proliferación celular para el mismo tiempo de cultivo que el grupo control. Algunos trabajos muestran evidencias de la acción específica del benceno sobre la cinética celular, ya por retraso del ciclo celular por exposición al benceno in vitro $(12,15,18)$ o por cambios en la cinética celular en individuos ocupacionalmente expuestos $(14,24)$ o reducción de la proliferación celular en ratones expuestos $(11,16)$. Otros au-tores no encuentran diferencias significativas (21-23). Los resultados sobre la cinética celular son importantes puesto que se sabe que algunos individuos expuestos crónicamente al benceno pueden desarrollar anemia aplástica o pancitopenia con disminución general de las líneas celulares hematopoyéticas y con un alto riesgo de desarrollar $\operatorname{LMA}(8,10,11)$. Sin embargo, en el presente trabajo no se tiene certeza sobre si los resultados de la cinética celular reflejan el efecto exclusivo de la exposición al benceno o a un conjunto de compuestos pues no todos los individuos están expuestos directamente al benceno, no todos mostraron alteración de la proliferación celular. En conclusión, el conjunto de resultados sobre la $\mathrm{XICH}$ y la cinética celular refleja el efecto sobre los cromosomas de la acción continua, crónica de posibles mutágenos carcinógenos ambientales derivados del petróleo, los cuales pueden alterar las características normales de la estructura y/o funcionamiento de los cromosomas y la posible alteración del funcionamiento celular.

\section{Agradecimientos}

Agradezco a Ecopetrol y sus trabajadores; y la colaboración técnica de Miriam Leivobici y Olga Moreno, de la Sección de Genética de la Universidad Nacional.

\section{Referencias}

1. Forni A, Pacífico E, Limonta A. Chromosome studies in workers exposed to benzene or toluene or both. Ach Env Health 1971; 22:373.

2. Bauchinger M, Schmid E, Dresp J, et al. Chromosome changes in lymphocytes after occupational exposure to toluene. Mutat Res 1982; 102:83.

3. Yarley-Jones A, Anderson D, Lovell D, Jenkinson P. Analysis of chromosomal aberrations in workers exposed to low level benzene. $\mathrm{Br} J$ Ind Med 1990; 47:48.

4. Yin S, Li G, Tain F, et al. Leukemia in benzene workers: a retrospective cohort study. Br J Ind Med 1987; 44:124.

5. Wong $\mathrm{O}$. An industry wide mortality study of chemical workers occupationally exposed to benzene I. General results. Br J Ind Med 1987; 44:365.

6. Aksoy M, Ozeris S, Sabuncu $\mathbf{H}$, et al. Exposure to benzene in Turkey between 1983 and 1985; a haematological study of 231 workers. Br J Ind Med 1987; 44:785.

7. Wongsrichanalai Ch, Delzell E, Cole P. Mortality from leukemia and other diseases among workers at a petroleum refinery. J Occ Med 1989; 31:106.

8. Golstein B. Benzene toxicity. J Occ Med 1988; 3:541.

9. Health and Safety Executive. Benzene Toxicity Review 1982; 4:1.

10. Rosen M, Snyder R. Protacted exposure to C57BL/6 mice to 300 ppm benzene depressed $B$ and T lymphocyte numbers and mitogen responses. Evidence for thymic and bone marrow proliferations in response to the exposure.Toxicology 1985; 37:13.

11. Maltoni C, Conti B, Cotti G, Belpoggi F. Experimental studies on benzene carcinogenicity at the Bologna Institute 
of Oncology: current results and the ongoing research. Am J Ind Med 1985; 7:415.

12. Morimoto K, WolfS. Increased sister chromatid exchanges and perturbations of cell division kinetics in human lymphocytes by benzene metabolites. Cancer Res 1980 ; 40:1189.

13. Witz G, Gad S, Tice R, et al . Genetic toxicity of the benzene metabolite trans, trans-muconalde-hyde in mammalian and bacterial cells. Mut Res 1990; 240:295.

14. Eastmond D, Smith M, Irons R. An interaction of benzene metabolites reproduces the myelotoxicity observed with benzene exposure. Toxicol and Applie Pharmacol 1987; 91:85.

15. Morimoto K. Induction of sister chromatid exchanges and cell cycle delay in human lymphocytes by microsomal activation of benzene. Cancer Res 1983; 43:1330.

16. Tice R, Costa D, Drew R. Cytogenetic effects of inhaled benzene in murine bone marrow: Induction of sister chromatid exchanges, chromosomeaberration and cellular proliferation inhibition in DBA/2 mice. Proc Natl Acad Sci USA 1980; 77:2148.

17. Salamanca F, Moreta G, Palma V, et al. Cytogenetic study in children chronically habituated to inhalation of volatile substances. Am J Med Genet 1987; 27:391.

18. Erexson G, Wilmer J, Kligerman A. Sister chromatid exchange induction in human lymphocytes exposed to benzene and its metabolites in vitro. Cancer Res 1985; 45:2471.

19. Watanabe T, Endo A, Kato Y, et al. Cytogenetics and citokinetics of cultured lymphocytes from benzene-exposed workers. Ind Arch Occup Env Health 1980; 46:311.

20. Sarto F, Cominato I, Pinton A, et al. A cytogenetic study on workers exposed to low concentrations of benzene. Carcinogenesis 1984; 5:827.

21. Clare G, Yarley A, Mclean A, Dean B. Chromosome analysis from peripheral blood lymphocytes of workers after acute exposure to benzene. $\mathrm{Br} \mathrm{J}$ Ind Med 1984; 41:249.

22. Yarley A, Anderson D, Jenkinson $P$, et al. Genotoxic effects in peripheral blood and urine of workers exposed to low level benzene. $\mathrm{Br} J$ Ind Med 1988; 45:694.

23. Seiji K, Jin Ch, Watanabe T, et al. Sister chromatid exchanges in peripheral lymphocytes of workers exposed to benzene, trichloroethylene, or tetrachloroethylene, with reference to smoking habits. Int Arch Env Health 1990; 62:171.

24. Skyberg K, Hansteen I, Jelmert O, Ronneberg A. A cytogenetic and haematological investigation of oil exposed workers in a Norwegian cable manufacturing company. $\mathrm{Br} \mathrm{J}$ Ind Med 1989; 46:791.

25. Perry $\mathbf{P}$, Wolf $\mathbf{S}$. New giemsa method for differential staining of sister chromatids. Nature 1974; 251:156.

26. Perry P, Evans H. Cytological detection of mutagencarcinogen exposure by sister chromatid exchange. Nature 1975; 258:121.

27. Tice R, Schneider S, Rary J. The utilization of bromodeoxiuridine incorporation into DNA for the analysis of cellular kinetics. Exp Cell Res 1976; 102:232.

28. Moorhead P, Novweel P, Mellam W, et al. Chromosome preparations of leukocytes cultures from peripheral blood. Exp Cell Res 1960; 20:613.

29. Hedner K, Hogster B, Kolning A, et al. Sister chromatid exchanges and structural aberrations in relation to 91 smoking individuals. Hereditas 1983; 98:77.

30. Hussum B, Wulf C, Neibuthr E. Sister chromatid exchange frequency correlates with age, sex and cigarrette smoking in a 5 year material of 553 healthy adults. Hereditas 1986; 105:17.

31. Kao-Shan C, Fina R, Whang-Peng J, et al. Increased fragile sites and sister chromatid exchanges in bone marrow and peripheral blood of young cigarrette smokers. Cancer Res 1987; 47:6.278.

32. Glover T, Stein C. Induction of sister chromatid exchanges at common fragile sites. Am J Hum Genet 1987; 41:882.

33. Yunis J, Soreng L, Bowe A. Fragile sites are targets of diverse mutagens and carcinogens. Oncogene 1987; $1: 59$.

34. Hetch F. The fragile hypothesis of cancer. Cancer Genet and Cytogenet 1988; 31:119.

35. Hetch F. Fragile sites, cancer chromosome breakpoints, and oncogenes all clustering light $G$ bands. Cancer Genet, and Cytogenet 1988; 31:17.

36. Wasvick H, Magnus P, Berg K. Effects of age, sex and genes on sister chromatid exchange. Clin Gene 1981; 20:449. 